\title{
Review on solar disturbance studies and electric power transmission line fault in Malaysia
}

\author{
Mohammad Zakaria Mohd Alias ${ }^{1}$, Mariyam Jamilah Homam², Faridah Hanim Mohd Noh ${ }^{3}$ \\ ${ }^{1,2}$ Wireless and Radio Science Centre, Faculty of Electrical and Electronic Engineering, \\ Universiti Tun Hussein Onn Malaysia, Malaysia \\ ${ }^{3}$ Faculty of Engineering Technology, Universiti Tun Hussein Onn Malaysia, Malaysia
}

\begin{tabular}{l} 
Article Info \\
\hline Article history: \\
Received Apr 10, 2019 \\
Revised Jul 14, 2019 \\
Accepted Jul 28, 2019 \\
\hline Keywords: \\
Digital fault recorder (DFR) \\
Geomagnetic induced current \\
(GIC) \\
Equatorial region \\
magnetometer \\
Solar disturbance \\
\hline
\end{tabular}

\begin{abstract}
This work is a review on previous research and studies on the solar disturbance activities and faults that occur on the electric power transmission line. Research has shown that low latitude regions could also be affected by solar disturbance events. The effects of these events on earth are considerable and may cause electric power transmission failure. Geomagnetically induced current is one of the ground impacts to the transmission line, which may cause fault or disturbance and consequently power system failure. Solar disturbance data from magnetometers and electric power transmission line fault data from a digital fault recorder are reviewed in this work. These data would be valuable in determining the characteristics and effects of solar disturbances on Malaysia's electric power transmission lines.
\end{abstract}

Copyright $@ 2020$ Institute of Advanced Engineering and Science. All rights reserved.

Corresponding Author:

Mariyam Jamilah Homam, Wireless and Radio Science Centre, Faculty of Electrical and Electronic Engineering, Universiti Tun Hussein Onn Malaysia, 86400 Parit Raja, Johor, Malaysia.

Email: mariyam@uthm.edu.my

\section{INTRODUCTION}

Space weather is a complex phenomenon that starts from the unstable conditions inside the sun and results in events such as coronal mass ejection (CME) or solar storm. CME can travel to space with tremendous speed that can reach the earth within 24 hours. Each blast of particles can pack the explosive energy of thousands or even millions of nuclear bombs. Another space weather phenomenon is called geomagnetic storms, which travel through interplanetary space and interact with Earth's space environment, which could possibly affect the earth ground systems, such as electric power system, telecommunication system, oil and gas pipelines and GPS system [1-5]. When these phenomena reach earth, they are called solar disturbances. The effects of these solar disturbance to the electric power system can cause critical situations. Power outages and electrical equipment failure caused by extreme CME in July 2012 were discussed in [6] and [7]. In [8], the effect was reported because a major blackout in occurred in three interconnected countries, from India's eastern border with Myanmar to its western border with Pakistan [8]. A study showed that the consequences of an extremely intense magnetic storm can result in a continental-scale failure of the electric power transmission line $[9,10]$. Previous studies have shown that solar disturbances may affect the low latitude countries [11]. In Malaysia, studies were conducted regarding the effects of solar disturbances [12-14]. In addition, several geomagnetic observatories have been installed in Malaysia in order to study the variations of geomagnetic field due to solar activity $[15,16]$.

The effects of solar disturbance that creates geomagnetic storms may affect electrical power transmission lines. Several studies were conducted regarding the geomagnetically induced current (GIC) on 
the electric power line [17-23]. Most of the studies were highlighting the high and middle latitude regions in which the power line are more prone to the solar disturbance. Meanwhile, only few studies focused at the low latitude region [24-26].

The effects of the solar disturbance on Malaysia's electric power transmission lines needs to be considered thoroughly. This is important because it contributes to the protection of Malaysia's electric power transmission lines from the uncertainty of solar disturbance event that might strike in the future. In this work, a review on previous research on the effects of solar disturbance to the electric power transmission line is presented. This research is a preliminary study to gather information on the previous studies on solar disturbances and electric power transmission line fault in Malaysia.

\section{SOLAR DISTURBANCE STUDY IN MALAYSIA}

Malaysia is situated close to the equator, and its climate is categorised as a tropical all throughout the year. The Space Environment Research Centre in Japan in collaboration with the Institute of Space Science (ANGKASA) and Universiti Kebangsaan Malaysia installed a magnetic data acquisition system (MAGDAS) magnetometer in Malaysia. Kyushu University in Japan initiated the installation of the MAGDAS sensor to study space weather. The first magnetometer was installed in Malaysia in September 2006 at the Langkawi National Observatory, Langkawi. The geographical coordinates of Langkawi station are $6^{\circ} 17^{\prime} \mathrm{N}, 99^{\circ} 46^{\prime} \mathrm{E}$. The location was selected based on two factors, its location near the magnetic equatorial region and low disturbance from human activity.

In [15] conducted a study in Peninsular Malaysia using a magnetometer, while in [16], the observation was performed in Sabah. In [15], observation of geomagnetic storms was carried out from June to December 2005 from a magnetic observatory station located in Parit Raja, Batu Pahat at $1^{\circ} 51.3^{\prime} \mathrm{N}$, $103^{\circ} 5.1^{\prime} \mathrm{E}$. The station consists of a fluxgate magnetometer used to measure the variations in magnetic components H, D and Z. The sampling rate is for $1 \mathrm{~s}$ and $1-\mathrm{min}$ Gaussian filter was applied to the data to produce 1-min data of $\mathrm{dH}, \mathrm{dD}$ and $\mathrm{dZ}$. The focus of the studies is on the CME effect. The southward component $(\mathrm{Bz})$ of the interplanetary magnetic field (IMF) is essential to the generation of a magnetic storm. The basic principle of a magnetic storm is the increase in ring current intensity, a circular current flowing around the earth on the equatorial line that produces a magnetic field disturbance. A global decrease in the horizontal component (H-component) of the geomagnetic field is observed at the ground level. The signature of a geomagnetic storm can be recognised when a huge reduction is observed in the H-components under the normal deviation measured by a magnetometer. The study successfully recorded nine geomagnetic storms during the seven-month observation period. The time duration of frequent magnetic storms was observed to be around the local noon 1400-2000 LT. The relation of the number of magnetic storms occurring on the day side to the night side is approximately $66 \%$. The magnetic activity records were derived from a network of near-equatorial geomagnetic observatories that measured the intensity of the globally symmetrical equatorial called the Dst index. By contrast, the daily regular magnetic field variation arising from the current systems caused by regular solar radiation changes is called the Kp index. The H-component variations compared with the global Kp and Dst indices showed a reasonable arrangement during geomagnetic storms.

In [16], the MAGDAS magnetometer was installed at Universiti Malaysia Sabah, Sabah. The magnetometer is located at $6^{\circ} 1^{\prime} \mathrm{N}, 116^{\circ} 4^{\prime} \mathrm{E}$. Geomagnetic events from 21 March 2013 to 26 March 2013 were recorded successfully by the magnetometer. Table 1 shows the locations of MAGDAS installed in Malaysia to monitor geomagnetic events.

Table 1. MAGDAS Magnetometer Sensor Locations in Malaysia

\begin{tabular}{ccc}
\hline International Code & Location & Area \\
\hline LKW & Langkawi National Observatory & North Malaysia \\
PER & Universiti Pendidikan Sultan Idris & North Malaysia \\
BTG & Agensi Angkasa Negara (Banting) & Center Malaysia \\
JOH & Universiti Teknologi Mara, Pasir Gudang & South Malaysia \\
TRE & Universiti Sultan Zainal Abidin & East Coast Malaysia \\
SBH & Universiti Malaysia Sabah & Borneo Island \\
\hline
\end{tabular}

In [11], studies on the CME effect that created geomagnetic disturbances during the quiet period were conducted by analysing a single CME event that occurred in 2014 and observing two parts, namely, space weather and geomagnetic data. Space weather data, such as solar wind speed (Vsw), northward interplanetary magnetic field (IMF Bz) and solar wind dynamic pressure (SW Pdyn), were taken from several conditions. The Vsw and IMF Bz were taken from the ACE satellite, data from which can be obtained from 
the Space Weather Prediction Centre website. The SW Pdyn can be calculated from the formula. The second part is from the geomagnetic data taken from five magnetometers, with four at the low latitude stations and one at the high latitude station, to determine the variations in the H-components at high and low latitudes. The study results show the CME affects the Vsw, IMF Bz and SW Pdyn. Studies also show that the variations in H-components at a low latitude stations with dayside stations are higher than the night ide one, which might be due to ionosphere convection. Development of space weather monitoring was conducted by a Malaysian researcher by creating a monitoring system website, the Space and Earth Electromagnetism Universiti Teknologi MARA (SEE-UiTM). This website monitoring system aims to provide information on solar activities to the satellite navigations and the power grid operator. SEE-UiTM is a compilation of many space weather websites to ensure easy, systematic and efficient monitoring, data collection and preparation of the report process [27].

The other area of solar disturbance studies in the Malaysia focuses on the effects of solar disturbance on the radio telecommunication system. In $[28,29]$, investigations on the implementation of realtime kinematic data were conducted to determine the ionosphere total electron content. The ionospheric layer is an important medium in the telecommunication system and consists of electrons, which are unpredictable due to time, season, solar activity, geomagnetic activity and latitude. In Malaysia, the various sources, such as telecommunication and navigation signals, of the manufactured radio frequency interference (RFI), are listed primarily by the Malaysian Communications and Multimedia Commission [30]. The ionosphere reflections are transmitted at very low frequency (VLF) with a frequency range of 3-30 $\mathrm{kHz}$ radio waves back to Earth. The strength of these radio waves is subject to the number or size of the ionised ionosphere. Solar radiation contributes to the ionisation of the atmosphere, which can alter the strength of the transmitted VLF radio waves [12]. Observations on the ionospheric disturbances caused by solar flare had been conducted in $[13,14]$. The study method includes analysing data from a continuously monitor and measures radio wave signal and the solar flare X-ray flux density. In [31], single solar flare events had been analysed in February 2011. In [17], the long solar flare events had been analysed from April 2013 to May 2013. Solar flare emissions cause substantial electron density variations, producing large amplitude and phase perturbations. Results indicated that the magnitudes of the perturbed signals are related directly to the power intensity of solar flare events. The intense X-ray fluxes can cause interference to the VLF signal. In [18], the effects of solar radiation on radio RFI were determined. The studies collect solar radiation data from the weather station located at the east coast of the Malaysian region and compared with the RFI. The data were examined with a spectrum analyser to determine the level of RFI. In the research, no obvious connection between solar radiation amount of energy received and the RFI pattern was observed, which may be due to several factors, such as observation time duration and absence of the low noise amplifier.

Previous studies have shown that research in Malaysia have focused on the observation and data collection on solar disturbances. Several geomagnetic storm events have been recorded successfully by previous studies, but the effects of geomagnetic storms still needs to be measured carefully, particularly its effects on Malaysia's electric power transmission lines. The effects of solar disturbances on the electric power transmission line needs to be considered to determine the occurrence of geomagnetically induced current in the electric power transmission line. This study is important because it contributes to the protection of Malaysia's electric power transmission lines from the uncertainty of solar disturbance event that might strike in the future.

\section{GEOMAGNETIC-INDUCED CURRENT}

The effects of solar disturbance that creates geomagnetic storms that can affect electrical power transmission lines involve a number of considerations, including GIC. Ground structures, such as power line, will create a path for the current flow that returns to the varying external currents in the high atmosphere. Once the natural current paths are accounted for, the net geoelectric field imposed on the artificial current paths results in a quasi-D.C. current in the power lines that can cause faults or disturbances to the power line. Previous studies have shown that GICs typically occur on high latitudes and the effects are highly eminent in the Northern regions due to sub/storm-time aurora electrojets. In [19], a detailed study of GIC occurrence, which caused a blackout in the Swedish network, was initiated. Studies concerning North American [20], African [21], Finnish [22] and UK-Scottish [23] power grids have also been found. Recent studies in [24] and [32] show that the amplification of the ring current can trigger large GICs at mid and low latitudes during storm sudden commencement.

The GIC calculation is important to determine the amplitude of current flows in the electric power transmission line when solar disturbance occurs. Such calculation has many methods as discussed in [32] where such process can be performed by several models. These models include plane wave, line current, $3 \mathrm{D}$ ionospheric-magnetospheric current system and complex image method plane wave model. The latter is a 
simple model for calculating the geoelectric field containing a plane wave primary field that propagates vertically downwards. The Earth is regarded as an unvarying half space (with conductivity $\sigma$ ). Considering a single angular frequency $\omega$, a horizontal electric field component Ey can be expressed in terms of the perpendicular horizontal magnetic field component $B x$ :

$$
E_{y}=-\sqrt{\frac{\omega}{\mu 0 \sigma}} e^{i \frac{x}{4}} B x
$$

where $\mu 0$ is the vacuum (and Earth) permeability. As shown in (1) believes the displacement currents can be deserted, which is normally adequate in geoelectromagnetics because the frequencies are very small $(\leq 1 \mathrm{~Hz})$. The ratio of the electric field to the magnetic field increases with an increasing frequency and a decreasing conductivity. As shown in (1) is known as the "basic equation of magnetotellurics" because it provides the basis for determining the Earth's conductivity by using electric (or telluric) and magnetic data recorded at the surface.

In [33] reported that GIC amplitudes at approximately $15 \mathrm{~A}$ and at a low latitude at $500 \mathrm{kV}$ power line had been recorded. This value is similar to the GIC recorded at high latitude. The location in the central territory of Brazil electric power line was selected. The measurement of GIC was obtained by installing a current sensor device at the neutral line of Itumbiara $500 \mathrm{kV}$ transformer from 2009 to 2013 . The LehtinenPirjola method was used in the GIC calculation. The other method of calculation has also been discussed in [34] by using the mesh impedance matrix method to calculate GIC with different network topology models and analyse the effects of the line length and network topology on GIC amplitudes. This method is proposed to consider the expected GIC in different networks and identify critical characteristics regarding GIC. In [33], overhead shield wire was considered during GIC calculation. The calculation method was divided into two parts. The first part is the geophysical one, which aims to determine the horizontal geoelectric field that consists of Earth's conductivity structure and ionospheric-magnetospheric currents or the magnetic field on the ground. The second part is the engineering one wherein GIC produced by the electric field is calculated. The network configuration and resistances constitute the input for part 2.

Previous research in Malaysia has also focused on GIC observation and measurement. In [25] and [35], the studies on GIC estimation were conducted for low latitude region by collecting data from space activity and magnetometer sensor. In [25], the time derivative of the horizontal component may be related directly to the GIC. Most effects to the low latitude are caused by the sudden impulse (SI). SI creates a large magnetic difference, which was detected by the magnetometer installed in Malaysia. Several factors, such as Vsw, Bz element of IMF and local time, had been discussed and measured to determine their contribution to the ground magnetic disturbance. During a sudden commencement (SC) with $\mathrm{dH} / \mathrm{dt}$ exceeding $30 \mathrm{nT} / \mathrm{min}$ event, the station located at the low latitude region may also be affected by the GIC occurrence. In [26], the latitudinal effect during the variation of solar wind parameters towards the GIC was examined. Solar wind parameters, geomagnetic indices and horizontal components of the geomagnetic field were taken as inputs. The analysis is performed on different latitudinal regions to study the latitudinal factor towards the GIC occurrence. The observation shows that the high $\mathrm{dH} / \mathrm{dt}$ value is obtained during the sudden increase of solar wind velocity associated with south-pointed IMF Bz. Simultaneously, the same high $\mathrm{dH} / \mathrm{dt}$ value was also recorded at the high latitudinal region.

Concerns on the effects of GIC to the electric power transmission line have opened up research opportunities to various countries, including Malaysia, located in the equatorial region. GIC studies in Malaysia have only been starting in recent years. Comparative studies need to be conducted across different latitudinal regions to understand the effects of the GIC occurrence. Results of previous research are essential in conducting GIC research in Malaysia. A large number of factors, such transmission line voltage level and location, valid information or data on the solar disturbance event and electrical utilities, need to be investigated thoroughly.

\section{FAULT ANALYSIS ON ELECTRIC POWER TRANSMISSION LINES}

IEEE C37.111 and IEC 60255-24 provide the format for records containing disturbance waveform and event data collected from power systems or power system models called communication transient data exchange (COMTRADE). Four files comprise the COMTRADE format namely, a configuration file (*.cfg), a data file (*.DAT), a header file (*.HDR) and an information file (*.INF). The *.DAT can be in ASCII or BINARY format, which contains real disturbance data of the power source, location and data format. The *.cfg consists of the voltage and current line profiles for each phase, frequency and version. The *.HDR, which is non-compulsory, can be used to provide information on the power source, location and data format. The *.INF is optional and can be used to provide setup information. 
The COMTRADE file is available in three versions. The common files are in 1991 and 1999 versions. The latest ones are in 2013 version. The digital fault recorder (DFR) is used to monitor, record and restore fault or disturbance event. Such equipment is also used to analyse protection operation, such as relay and circuit breaker. DFR can monitor the entire substation by configuring the number of data acquisition modules of the substation. DFR also has high recording sampling frequency at a higher range $(5 \mathrm{kHz})$ compared with a protective relay $(1 \mathrm{kHz})$. DFR records the instant values (waveforms) of the current and voltages, which may be sampled many times per cycle [36-38].

Malaysia is located at the equatorial region, which is subject to two monsoon seasons. Lightning strike is one of the frequent causes of tripping, which results in faults to the Malaysian electric power transmission lines. Other faults include tree encroachment, animal and crane encroachment and equipment failure [39]. The difference between DFR and IED is the various sampling rates. DFR has more sampling rate that the IED. Engineers from Tenaga Nasional Berhad, which is the main electric utility company in Malaysia, conducted a study on the DFR application in the different voltage levels of the transmission line. The DFR is used to record an actual disturbance in the transmission line. The research focused on the analysis of the fault, protection equipment operation and the improvements that might be applied. The fault analysis, which covered fault duration, protection relay sequence and circuit breaker events, is based on the COMTRADE file retrieved from the DFR [40].

The power protection system's consistency and discrimination have become critical issues and the development of numerical protection technology contributes to the convolution of the protection system investigation. Therefore, a DFR is vital to the investigation of the system protection. One advantage of such equipment is it checks the accuracy of protection operation compared with normal utility practice [41, 42]. Several studies have discussed the protection system performance information to identify the faulty section and confirm the protection operations. In [43] discussed the combination of proficient and model-based systems to categorise protection accuracy. The study focused on accurate protection process arrangements and modelling of any given disturbance that occurs in the electric power transmission line. The model must be updated to the latest power system status to perform accurate identification. Faults or disturbances can be captured through protection relay and DFR and stored in the local management computer placed at the substation. Traditionally, such data are collected manually. With internet connection, these data are sent to the control room for the operator to make a decision. This approach requires that the aforementioned protective relays be upgraded to digital ones.

Power system protection and monitoring must be fast and dependable. Fast fault clearing time is crucial because of various concerns, such as protection system malfunction and reduction of the effects of any system fault, which may cause power system failure or outages [44]. Such condition may lead to protection performance inspection and monitoring for each operation becoming a routine. By contrast, the inability to notice protection failures prior to any system incident can lead to equipment malfunction. Protection system analysis requires massive knowledge and comprehensive understanding of the protection system function. Therefore, monitoring of all protection operations as a backup system must be conducted and coordinated to detect any failure in the protection system and address future fault events [45]. Numerous methods for transmission line fault detection and classification are already available using various techniques, including artificial neural network [46, 47] and wavelet transform [48].

In [49], a new method for fault classification using a transient monitoring function was proposed. The method is more accurate as compared to the discrete wavelet transform method. The algorithm required several considerations, such as line current, fault location, inception angle, fault resistance and load angle. Fault classification is important in the protection system to provide accurate information for protection relay operation. DFR is a useful equipment to provide the actual transmission line fault or disturbance records. The COMTRADE file produced by the DFR is important in investigating the fault in the transmission line. Such file may provide information on the fault time and duration, transmission line location, voltage and current variation, harmonics and operation information protection. This file is important in determining the characterisations of solar disturbance effects on the electric power system network.

\section{CONCLUSION}

This paper presents a review on the solar disturbance events and the electric power transmission line fault. Based on the previous researches, most of the studies were conducted at the middle and high latitude regions, and only a few at the low latitude region. The effects of solar disturbances on Malaysia's electric power transmission lines have not been studied in detail. Hence, our research aims to investigate the effects of solar disturbances on Malaysia's electric power transmission lines by gathering data from a magnetometer sensor and digital fault recorder. The potential for use of these data in determining the occurrences of geomagnetic events that create geomagnetically induced current should be explored. Studies on the effect of 
solar disturbance are important to protect and maintain Malaysia's electric power transmission lines. Our research could usher new and innovative research areas in electrical power system studies and inspire students, researchers and electrical engineers in Malaysia.

\section{ACKNOWLEDGEMENT}

The authors would like to express their gratitude to the Ministry of Education Malaysia for funding this project through FRGS Vot 1628 research grant.

\section{REFERENCES}

[1] A. Pulkinnen, "Geomagnetic Induction during Highly Disturbed Space Weather Conditions: Studies of Ground Effects", Academic Dissertation, University of Helsinki, Faculty of Science, Department of Physical Sciences, 2003.

[2] R. Pirjola, "Space weather effects on power grids, in: Space Weather- Physics and Effects," Springer Praxis Books, Springer Berlin Heidelberg, 2007.

[3] D.H Boteler et al., "The Effects of Geomagnetic Disturbances on Electrical Systems at the Earth's Surface", Advances in Space Research, 22(1), pp. 17-27, 1998.

[4] R. Pirjola, "Geomagnetically Induced Currents as Ground Effects of Space Weather", in Space Science, 2012.

[5] D. H. Boteler, "Geomagnetic Hazards to Conducting Networks", Natural Hazards, 28: pp. 537-561, 2003.

[6] T. Phillips, "Near Miss: The Solar Super storm of July 2012", https://science.nasa.gov/science-news/science-atnasa/2014/23jul_superstorm,13.04.2019, 2014.

[7] D. L. Ying et al., "Observations of an extreme storm in interplanetary space caused by successive coronal mass ejections", Nature communications. 5, 3481, 2014.

[8] Y. Jim and H. Gardiner, "2nd Day of Power Failures Cripples Wide Swath of India, 31 July 2012", https://willblogforfood.typepad.com/will_blog_for_food/2012/07/2nd-day-of-power-failures-cripples-wide-swathof-india.html, 2012.

[9] CENTRA Technology, Inc., "Geomagnetic Storms, Office of Risk Management and Analysis", United States Department of Homeland Security, https://www.oecd.org/gov/risk/46891645.pdf, 2011.

[10] National Research Council, "Severe Space Weather Events - Understanding Societal and Economic Impacts", A Workshop Report, 2008.

[11] F. A. M. Kasran et al., "Geomagnetic disturbance during quiet period at low-latitude stations," 2015 International Conference on Space Science and Communication (IconSpace), 2015, pp. 273-276.

[12] M. M. A. Rashid et al., "VLF observation of D-region disturbances associated with solar flares at UKM Selangor Malaysia", 2013 IEEE International Conference on Space Science and Communication (IconSpace) 2013, pp. 249-252.

[13] H. Y. Hwa et al., "Equatorial TEC variations during the Geomagnetic Storm of July 15-17, 2000", in General Assembly Union Radio Science International, 17-24 August 2002, Maastricht, Netherlands.

[14] M. M. A. Rashid et al., "Ionospheric disturbances triggered by solar flare events during solar maximum — May 2013”, 2015 IEEE 12th Malaysia International Conference on Communications (MICC), 2015, pp. 192-196.

[15] A. F. M. Zain and M. K. Othman, "Observations of magnetic storms at the newly developed magnetic observatory in Malaysia", International Conference on Science and Technology (ICSTIE '06), 8-9 December 2006.

[16] S. N. Ibrahim et al., "First Geomagnetic Observation at Sabah, Malaysia by using MAGDAS Array", International Journal of Simulation: Systems, Science and Technology, 17 (41), pp. 30.1-30.8., 2017.

[17] A. Pulkkinen et al., "Geomagnetic storm of 29-31 October 2003: Geomagnetically induced currents and their relation to problems in the Swedish high-voltage power transmission system", Space Weather, 3(8), pp. 1-19, 2005.

[18] L. H. Wei et al., "Surface electric fields for North America during historical geomagnetic storms", Space Weather, 11(8), pp. 451-462, 2013.

[19] J. Koen and C.T. Gaunt, "Disturbance in the Southern African power network due to geomagnetically induced currents", Cigre Session, Paris, Paper 36-206, 2002.

[20] A. Viljanen and R. Pirjola, "Geomagnetically induced currents in the Finnish high-voltage power system", Surv Geophys, 15: 383, 1994.

[21] D. Beamish et al., "Geomagnetically induced currents in the UK: Geomagnetic variations and surface electric fields", Journal of Atmospheric and Solar-Terrestrial Physics, 64(16), pp. 1779-1792, 2002.

[22] S. Watari et al., "Measurements of geomagnetically induced current in a power grid in Hokkaido, Japan", Space Weather, vol. 7, no. 3, pp. 1-11, 2009.

[23] C. Barbosa et al., "Analysis of geomagnetically induced currents at a low-latitude region over the solar cycles 23 and 24: Comparison between measurements and calculations", Journal of Space Weather and Space Climate, Vol. 5, 2015.

[24] N.B. Trivedi et al., "Geomagnetically induced currents in an electric power transmission system at low latitudes in Brazil: A case study", Space Weather, 5(4), 2007.

[25] F. M. Kasran et al., "The time derivative of the horizontal geomagnetic field for the low latitude MAGDAS Langkawi station for the estimation of geomagnetically induced current," In: Suparta W., Abdullah M., Ismail M. (eds) Space Science and Communication for Sustainability. Springer, Singapore, 2018. 
[26] Z. I. A. Latiff et al., "Latitudinal investigation on the variation of solar wind parameters towards geomagnetically induced currents during 7-8 September 2017 disturbed period", 2018 IEEE 8th International Conference on System Engineering and Technology (ICSET 2018), pp. 123-127.

[27] N. Z. Jusoh et al., "Development of space weather monitoring platform for space and earth electromagnetism observation", Journal of Engineering and Applied Sciences, 12(10), pp. 3308-3311, 2017.

[28] H. M. N. Hanif et al., "Implementation of Real-Time Kinematic Data to Determine the Ionospheric Total Electron Content", 2012 Third International Conference on Intelligent Systems Modelling and Simulation, 2012, pp. 238-243.

[29] A. F. M. Zain and M. K. Othman, "Initial Program Towards Developing Magnetic Observatory in Malaysia", Proceeding of XI the IAGA Workshop on Geomagnetic Observatory Instruments, Data Acquisition and Processing, Tsukuba, Japan, November 9 - 17, 2004.

[30] Z. Z. Abidin et al., "RFI profiles of prime candidate sites for the first radio astronomical telescope in Malaysia", New Astronomy, 15(3), pp. 307-312, 2010.

[31] N. H. Sabri et al., "The effect of solar radiation on radio signal for radio astronomy purpose", Malaysian Journal of Analytical Sciences, Vol 19 No 6, pp. 1374 - 1381, 2015.

[32] P. Pirjola, "Review on the calculation of surface electric and magnetic fields and geomagnetically induced currents in ground based technological systems", Surveys in Geophysics, 23(1), pp. 71-90, 2002.

[33] R. Pirjola, "Calculation of geomagnetically induced currents (GIC) in a high-voltage electric power transmission system and estimation of effects of overhead shield wires on GIC modeling", Journal of Atmospheric and SolarTerrestrial Physics, 69(12), pp. 1305-1311, 2007.

[34] K. Zheng et al., "Calculation analysis of geomagnetically induced currents with different network topologies," 2013 IEEE Power \& Energy Society General Meeting, pp. 1-4.

[35] N. M. Anuar et al., "The behavior of solar wind parameters and geomagnetic activity indices for geomagnetically induced current observations", Journal of Fundamental and Applied Sciences, 20(1), 2018.

[36] IEEE Standard Common Format for Transient Data Exchange (COMTRADE) for Power Systems, in IEEE Std C37.111-1999, pp.1-55.

[37] IEEE/IEC Measuring relays and protection equipment - Part 24: Common format for transient data exchange (COMTRADE) for power systems, in IEEE Std C37.111-2013 (IEC 60255-24 Edition 2.0 2013-04), pp.1-73.

[38] A. A. M. Zain and S. A. P. Karim, "Protection system analysis using fault signatures in Malaysia", International Journal of Electrical Power \& Energy Systems, 45(1), pp. 194-205, 2013.

[39] M. Z. Alias and M. S. Shokri, "The design of IEC 61850 based Disturbance and Fault Recorder for online fault analysis in the System Verification and Simulation Laboratory”, 2015 International Symposium on Technology Management and Emerging Technologies (ISTMET), pp. 123-127.

[40] A. T. S. Chun and S. P. A. Karim, "Experience on Protection System Analysis Utilizing Fault Recorders and Numerical Relay Recordings”, 2009.

[41] H. Eristi and Y. Demir, "Automatic classification of power quality events and disturbances using the wavelet transform and support vector machines", in Generation, Transmission \& Distribution, IET, vol.6, no.10, pp.968-976, 2012.

[42] J. Morais et al., "A Framework for Evaluating Automatic Classification of Underlying Causes of Disturbances and Its Application to Short-Circuit Faults", in IEEE Transactions on Power Delivery, vol.25, no.4, pp.2083-2094, 2010.

[43] M. Kezunovic et al., IEEE - Tutorial on automated fault analysis, 2001.

[44] D. Saxena et al., "Power quality event classification: an overview and key issues", International Journal of Engineering, Science and Technology, vol. 2, no. 3, pp. 186-199, 2010.

[45] IEEE Committee Report, "Application and Evaluation of Automatic Fault-Recording Devices", in IEEE Transactions on Power Apparatus and Systems, vol. 84, no. 12, pp. 1187-1203, 1965.

[46] J. Majid et al., "Fault detection and classification in electrical power transmission system using artificial neural network", SpringerPlus, vol. 4 334, 2015.

[47] A. Azriyenni et al., "Backpropagation Neural Network Modeling for Fault Location in Transmission Line 150 kV", Indonesian Journal of Electrical Engineering and Informatics (IJEEI), Vol. 2, No. 1, pp. 1-12, 2014.

[48] N. Ghaffarzadeh, "A New Method for Recognition of Arcing Faults in Transmission Lines using Wavelet Transform and Correlation Coefficient", Indonesian Journal of Electrical Engineering and Informatics (IJEEI), Vol.1, No.1, pp. 1-7, 2013.

[49] P. R. Pattanaik et al., "Transient Monitoring Function based Fault Classifier for Relaying Applications", International Journal of Electrical and Computer Engineering (IJECE), vol. 8, no. 6, pp. 4089-4095, 2018. 


\section{BIOGRAPHIES OF AUTHORS}

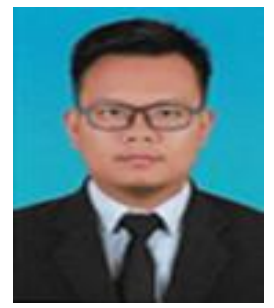

Mohammad Zakaria Mohd Alias received his Bachelor's degree from Universiti Teknikal Malaysia Melaka in 2010 and Master's degree from Universiti Malaya in 2014. He is currently a $\mathrm{PhD}$ student at the Faculty of Electrical and Electronic Engineering, Universiti Tun Hussein Onn Malaysia.

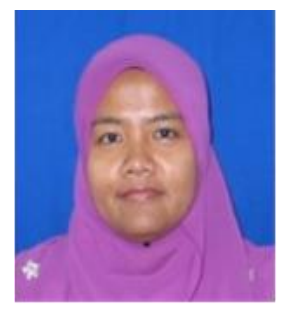

Mariyam Jamilah Homam is a Senior Lecturer at the Faculty of Electrical and Electronic Engineering, Universiti Tun Hussein Onn Malaysia. She received her Bachelor's and Master's degrees in Communication and Computer Engineering from Universiti Kebangsaan Malaysia in 2000 and 2003, respectively. She obtained her PhD from University of Leicester, UK in 2012.

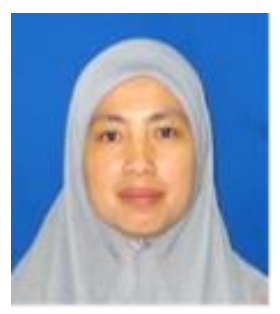

Faridah Hanim Mohd Noh received her Bachelor's degree from Universiti Malaya in 2004, Master's degree from Universiti Tun Hussein Onn Malaysia in 2008, and PhD in Kumamoto University in 2016. She is currently a Senior Lecturer at the Faculty of Engineering Technology, Universiti Tun Hussein Onn Malaysia. 